\title{
Multiple unbounded solutions for a boundary value problem on infinite intervals
}

\author{
Hairong Lian* and Fengjie Geng
}

* Correspondence: lianhr@126.com School of Information Engineering, China University of Geoscience, Beijing 100083, People's Republic of China

\begin{abstract}
This paper is concerned with the existence of multiple unbounded solutions for a Sturm-Liouville boundary value problem on the half-line. By assuming the existence of two pairs of unbounded upper and lower solutions, the existence of at least three solutions is obtained using the degree theories. Nagumo condition plays an important role in the nonlinear term involved in the first-order derivative. It is an interesting point that the method of unbounded upper and lower solutions is extended to obtain conditions for the existence of multiple solutions.

Mathematics Subject Classification (2000 34B10, 34B40
\end{abstract}

Keywords: infinite interval problem, multiplicity, unbounded upper solutions, unbounded lower solutions, degree theory

\section{Introduction}

In this paper, we will employ the method of unbounded upper and lower solutions to study the existence of Sturm-Liouville boundary value problem on the half-line

$$
\left\{\begin{array}{l}
u^{\prime \prime}(t)+\phi(t) f\left(t, u(t), u^{\prime}(t)\right)=0, \quad t \in(0,+\infty) \\
u(0)-a u^{\prime}(0)=B, \quad u^{\prime}(+\infty)=C
\end{array}\right.
$$

where $\varphi:(0,+\infty) \rightarrow(0,+\infty), f:[0,+\infty) \times \mathbb{R}^{2} \rightarrow \mathbb{R}$ are continuous, $a>0, B, C \in \mathbb{R}$.

The method of upper and lower solutions is a powerful tool to prove the existence of differential equation subject to certain boundary conditions. It is well known that nonlinear problems always have at least one solution in the ordered interval defined by one pair of well-ordered upper and lower solutions. To show this kind of result, we can employ the topological degree theory or monotone iterative technique, etc, see [1-5] and the reference therein.

Boundary value problems to differential equations on the half-line arise naturally in the study of radially symmetric solutions of nonlinear elliptic equations, and many works have been done in this area, see [6]. When applying the method of upper and lower solution method to discuss the infinite interval problem, most of the results are concerned with the existence of the bounded or positive solutions. Chen and Zhang [7], with $a=0$, discussed the lower and upper solution technique and presented the existence of positive solutions with sublinearity conditions. In [8], Agarwal and O'Regan studied the equation $(1 / p)\left(p y^{\prime}\right)^{\prime}=q(t) f(t, y)$ with the boundary condition $y(t)$ bounded on $[0,+\infty)$ or $\lim _{t \rightarrow+\infty} y(t)=0$ using the upper and lower solution technique.

(c) 2011 Lian and Geng; licensee Springer. This is an Open Access article distributed under the terms of the Creative Commons Attribution License (http://creativecommons.org/licenses/by/2.0), which permits unrestricted use, distribution, and reproduction in any medium, provided the original work is properly cited. 
The methods used therein were based on diagonalization arguments and existence results of appropriate boundary value problems on finite intervals using upper and lower solutions. In [9], Palamides and Galanis proved the existence of a global, monotone, positive and unbounded solution. Recently, Yan, Agarwal and O'Regan [10], with $C>0$ in BVP (1), presented sufficient conditions for the existence of unbounded positive solutions. In [11], the authors established further the unbounded upper and lower theory for such boundary value problem. Using such technique coupled with the Schäuder fixed point theorem, the existence of the unbounded solution is obtained.

How many solutions exist when infinite interval nonlinear problem has two pairs of well-ordered lower and upper solutions? Eloe, Kaufmann and Tisdell [12] have presented some sufficient conditions for the existence of three solutions for the equation $x^{\prime \prime}-a(t) x+f(t, x)=0$ with the boundary conditions $x(0)=x_{0}$ and $x(t)$ bounded on [0, $+\infty$ ) by employing the degree theory on compact domains and the sequential arguments on unbounded domain. But, to the best knowledge of the authors, when considering the upper and lower solution method, there is no paper to find the existence of multiple unbounded solutions for infinite interval problem.

Inspirited by the papers mentioned above, in this paper, we aim to use the upper and lower solution method to discuss the existence of multiple unbounded solutions for infinite interval problem. For BVP (1), by assuming two unbounded lower solutions $\alpha_{1}$, $\alpha_{2}$ and two unbounded upper solutions $\beta_{1}, \beta_{2}$ satisfying $\alpha_{1} \leq \alpha_{2}, \beta_{1} \leq \beta_{2}, \alpha_{2} \otimes \beta_{1}$, the Nagumo condition and additional suitable ones on $f$ to yield prior bounds of $u$ and $u$, we show that the infinite interval problem (1) has at least three solutions. The bases of the degree theory and the truncations analysis are exposed in this paper, which are somewhat different from those in [12]. The solutions obtained in this paper are admitted to be unbounded. And the extension of the unbounded upper and lower solution method to obtain conditions for the existence of multiple solutions is interesting.

\section{Definitions}

In this section, we present the definition of unbounded upper solutions, unbounded lower solutions, Nagumo condition and a special Banach space.

Definition 2.1. A function $\alpha \in C^{1}[0,+\infty) \cap C^{2}(0,+\infty)$ is called a lower solution of BVP (1) if

$$
\left\{\begin{array}{l}
\alpha^{\prime \prime}(t)+\phi(t) f\left(t, \alpha(t), \alpha^{\prime}(t)\right) \geq 0, \quad t \in(0,+\infty) \\
\alpha(0)-a \alpha^{\prime}(0) \leq B, \quad \alpha^{\prime}(+\infty)<C
\end{array}\right.
$$

A function $\beta \in C^{1}[0,+\infty) \cap C^{2}(0,+\infty)$ is called an upper solution of BVP (1) if

$$
\left\{\begin{array}{l}
\beta^{\prime \prime}(t)+\phi(t) f\left(t, \beta(t), \beta^{\prime}(t)\right) \leq 0, \quad t \in(0,+\infty) \\
\beta(0)-a \beta^{\prime}(0) \geq B, \quad \beta^{\prime}(+\infty)>C
\end{array}\right.
$$

A function $\alpha \in C^{1}[0,+\infty) \cap C^{2}(0,+\infty)$ is called a strict lower solution of BVP (1) if the inequality in (2) is strict for $t \in(0,+\infty)$. A strict upper solution is defined similarly.

Remark 2.1. Since the continuous functions $\alpha, \beta$ are defined on $[0,+\infty)$, they are admitted to be unbounded. So, we call them unbounded lower and upper solutions.

Definition 2.2. Given a pair of functions $\alpha, \beta \in C^{1}[0,+\infty)$ satisfying $\alpha(t) \leq \beta(t), t \in$ $[0,+\infty)$. A function $f:[0,+\infty) \times R^{2} \rightarrow R$ is said to satisfy Nagumo condition with 
respect to the pair of functions $\alpha, \beta$, if there exists a nonnegative function $\psi \in C[0$, $+\infty)$ and a positive one $h \in C[0,+\infty)$ such that

$$
|f(t, x, y)| \leq \psi(t) h(|\gamma|)
$$

for all $0 \leq t<+\infty, \alpha(t) \leq x \leq \beta(t)$ and

$$
\int_{0}^{+\infty} \psi(s) \phi(s) \mathrm{d} s<+\infty, \quad \int^{+\infty} \frac{s}{h(s)} \mathrm{d} s=+\infty .
$$

Consider the space $X$ defined by

$$
X=\left\{x \in C^{1}[0,+\infty), \quad \lim _{t \rightarrow+\infty} x^{\prime}(t) \text { exists }\right\},
$$

with the norm $\|x\|=\max \left\{|| x\left\|_{1},\right\| x^{\prime}||_{\infty}\right\}$, where $\|x\|_{1}=\sup _{t \in[0,+\infty)}\left|\frac{x(t)}{1+t}\right|,\left\|x^{\prime}\right\|_{\infty}=$ $\sup _{t \in[0,+\infty)}\left|x^{\prime}(t)\right|$. By the standard arguments, we can prove that $(X,\|\cdot\|)$ is a Banach space.

Remark 2.2. Banach space $X$ is introduced in order to estimate the prior bound of the solutions to BVP (1), which is necessary to apply the degree theory. The solution of BVP (1) is bounded with the norm $\|\cdot\|$ in $X$ as well as the upper and lower solutions, but they are not as a continuous function.

\section{Existence of solutions}

For the sake of convenience, we list the assumptions to be used in this section.

$\left(\mathrm{H}_{1}\right)$ BVP (1) has two pairs of upper-lower solutions $\beta_{i}, \alpha_{i} \in X, i=1$, 2 satisfying

$$
\alpha_{1}(t) \leq \alpha_{2}(t) \leq \beta_{2}(t), \alpha_{1}(t) \leq \beta_{1}(t) \leq \beta_{2}(t), \alpha_{2}(t) \not \leq \beta_{1}(t), t \in[0,+\infty),
$$

where $\alpha_{2}, \beta_{1}$ are strict lower and upper solutions, respectively.

$\left(\mathrm{H}_{2}\right) f$ satisfies Nagumo condition with respect to $\alpha_{1}, \beta_{2} . \varphi \in L^{1}[0,+\infty)$ and there exists $\gamma>1$ such that $\sup _{0 \leq t<+\infty}(1+t)^{\gamma} \phi(t) \psi(t)<+\infty$.

$\left(\mathrm{H}_{3}\right)$ For any $r>0$, there exists $\phi_{r}$ such that for $0 \leq t<+\infty, \alpha_{1}(t) \leq x \leq \beta_{2}(t), 0 \leq y \leq$ $r$, we have

$$
f(t, x, y) \leq \varphi_{r}(t) \text { and } \int_{0}^{+\infty} \phi(s) \varphi_{r}(s) \mathrm{d} s<+\infty .
$$

Theorem 3.1. Suppose conditions $\left(\mathrm{H}_{1}\right)$ and $\left(\mathrm{H}_{2}\right)$ hold. Then, $B V P(1)$ has at least three solutions $u_{1}, u_{2}, u_{3}$ satisfying

$$
\alpha_{1} \leq u_{1} \leq \beta_{1}, \alpha_{2} \leq u_{2} \leq \beta_{2}, u_{3} \not \leq \beta_{1}, u_{3} \nsucceq \alpha_{2} .
$$

Proof. Choose $R>C$,

$$
\eta \geq \max \left\{\sup _{t \in[\delta,+\infty)} \frac{\beta_{2}(t)-\alpha_{1}(0)}{t}, \sup _{t \in[\delta,+\infty)} \frac{\beta_{2}(0)-\alpha_{1}(t)}{t}\right\}
$$


with $\delta>0$ a certain constant such that

$$
\int_{\eta}^{R} \frac{s}{h(s)} \mathrm{d} s \geq M\left(\sup _{t \in[0,+\infty)} \frac{\beta_{2}(t)}{(1+t)^{\gamma}}-\inf _{t \in[0,+\infty)} \frac{\alpha_{1}(t)}{(1+t)^{\gamma}}+\frac{\gamma}{\gamma-1} \cdot \sup _{t \in[0,+\infty)} \frac{\beta_{2}(t)}{1+t}\right)
$$

where $M=\sup _{0 \leq t<+\infty}(1+t)^{\gamma} \phi(t) \psi(t)$. And consider the truncated boundary value problem

$$
\left\{\begin{array}{l}
u^{\prime \prime}(t)+\phi(t) f_{1}^{*}\left(t, u(t), u^{\prime}(t)\right)=0, \quad t \in(0,+\infty) \\
u(0)-a u^{\prime}(0)=B, \quad u^{\prime}(+\infty)=C
\end{array}\right.
$$

where

$$
f_{1}^{*}(t, x, y)= \begin{cases}f_{R}\left(t, \alpha_{1}(t), y\right)+\frac{\alpha_{1}(t)-x}{1+\left|x-\alpha_{1}(t)\right|}, & x<\alpha_{1}(t) \\ f_{R}(t, x, y) & \alpha_{1}(t) \leq x \leq \beta_{1}(t) \\ f_{R}\left(t, \beta_{2}(t), y\right)+\frac{\beta_{2}(t)-x}{1+\left|x-\beta_{2}(t)\right|}, & x>\beta_{2}(t)\end{cases}
$$

and

$$
f_{R}(t, x, y)=\left\{\begin{array}{l}
f(t, x,-R), y<-R \\
f(t, x, y), \quad-R \leq y \leq R \\
f(t, x, R), \quad y>R
\end{array}\right.
$$

Obviously, if (7) has triple solutions $u_{i}, i=1,2,3$ satisfying $\alpha_{1} \leq u_{i} \leq \beta_{2}$ and $\left\|u_{i}^{\prime}\right\|_{\infty}<R$, then we can complete the proof. Next, we will prove sequentially that if $u$ is a solution of (7), it holds $\alpha_{1} \leq u \leq \beta_{2},\left\|u^{\prime}\right\|_{\infty}<R$ and (7) has at least three solutions.

Step 1: If $u$ is a solution of (7), it holds $\alpha_{1}(t) \leq u(t) \leq \beta_{2}(t), t \in[0,+\infty)$.

We just show $u(t) \leq \beta_{2}(t), t \in[0,+\infty)$. If it does not hold, we have

$$
\sup _{0 \leq t<+\infty}\left(u(t)-\beta_{2}(t)\right)>0 .
$$

Because $u^{\prime}(+\infty)-\beta_{2}^{\prime}(+\infty)<0$, so there are two cases.

Case 1. $\lim _{t \rightarrow 0^{+}}\left(u(t)-\beta_{2}(t)\right)=\sup _{0 \leq t<+\infty}\left(u(t)-\beta_{2}(t)\right)>0$.

Easily, it holds $u^{\prime}\left(0^{+}\right)-\beta_{2}^{\prime}\left(0^{+}\right) \leq 0$. While from the boundary condition, we have

$$
u^{\prime}(0)-\beta_{2}^{\prime}(0) \geq \frac{1}{a}\left(u(0)-\beta_{2}(0)\right)>0,
$$

which is a contraction.

Case 2. There exists $t^{*} \in(0,+\infty)$ such that

$$
u\left(t^{*}\right)-\beta_{2}\left(t^{*}\right)=\sup _{0 \leq t<+\infty}\left(u(t)-\beta_{2}(t)\right)>0 .
$$

So we have $u^{\prime}\left(t^{*}\right)-\beta_{2}^{\prime}\left(t^{*}\right)=0, u^{\prime \prime}\left(t^{*}\right)-\beta_{2}^{\prime \prime}\left(t^{*}\right) \leq 0$. Unfortunately,

$$
\begin{aligned}
& u^{\prime \prime}\left(t^{*}\right)-\beta_{2}^{\prime \prime}\left(t^{*}\right) \\
& \geq \phi\left(t^{*}\right)\left(f\left(t^{*}, \beta_{2}\left(t^{*}\right), \beta_{2}^{\prime}\left(t^{*}\right)\right)-f_{1}^{*}\left(t^{*}, u\left(t^{*}\right), u^{\prime}\left(t^{*}\right)\right)\right) \\
&=\phi\left(t^{*}\right) \frac{u\left(t^{*}\right)-\beta_{2}\left(t^{*}\right)}{1+\left|u\left(t^{*}\right)-\beta_{2}\left(t^{*}\right)\right|} \\
&>0 .
\end{aligned}
$$


Which is also a contraction. Here, we note that $\left\|\beta_{2}\right\|_{\infty}<R$ holds with similar discussions to those in step 2.

Consequently, $u(t) \leq \beta_{2}(t)$ holds for all $t \in[0,+\infty)$. Similarly, we can show that $\alpha_{1}(t)$ $\leq u(t)$ for all $t \in[0,+\infty)$.

Step 2: If $u$ is a solution of (7), then $\left\|u^{\prime}\right\|_{\infty} \leq R$.

From step 1 , we know that $\alpha_{1} \leq u \leq \beta_{2}$ if $u$ is a solution of (7). If $\left\|u^{\prime}\right\|_{\infty} \leq R$ is untrue, we have the following three cases.

Case 1. $\left|u^{\prime}(t)\right|>\eta, \forall t \in[0,+\infty)$.

Without loss of generality, we suppose $u^{\prime}(t)>\eta, t \in[0,+\infty)$. While for any $T \geq \delta$,

$$
\frac{\beta_{2}(T)-\alpha_{1}(0)}{T} \geq \frac{u(T)-u(0)}{T}=\frac{1}{T} \int_{0}^{T} u^{\prime}(s) \mathrm{d} s>\eta \geq \frac{\beta_{2}(T)-\alpha_{1}(0)}{T}
$$

which is a contraction. So, there must exist $t_{0} \in[0,+\infty)$ such that $\left|u^{\prime}\left(t_{0}\right)\right| \leq \eta$.

Case 2. $\left|u^{\prime}(t)\right| \leq \eta, \forall t \in[0,+\infty)$.

Just take $R$ such as the definition of $\eta$ in (6) and we can complete the proof.

Case 3. There exists $\left[t_{1}, t_{2}\right] \subset[0,+\infty)$ such that $\left|u^{\prime}\left(t_{1}\right)\right|=\eta,\left|u^{\prime}(t)\right|>\eta, t \in\left(t_{1}, t_{2}\right]$ or $\mid$ $u^{\prime}\left(t_{2}\right)|=\eta,| u^{\prime}(t) \mid>\eta, t \in\left[t_{1}, t_{2}\right)$.

Without loss of generality, we suppose that $u^{\prime}\left(t_{1}\right)=\eta$, $u^{\prime}(t)>\eta, t \in\left(t_{1}, t_{2}\right]$. Obviously,

$$
\begin{aligned}
& \int_{u^{\prime}\left(t_{1}\right)}^{u^{\prime}\left(t_{2}\right)} \frac{s}{h(s)} \mathrm{d} s=\int_{t_{1}}^{t_{2}} \frac{u^{\prime}(s)}{h\left(u^{\prime}(s)\right)} u^{\prime \prime}(s) \mathrm{d} s \\
& =\int_{t_{1}}^{t_{2}} \frac{-\phi(s) f_{1}^{*}\left(s, u(s), u^{\prime}(s)\right) u^{\prime}(s)}{h\left(u^{\prime}(s)\right)} \mathrm{d} s \\
& \leq \int_{t_{1}}^{t_{2}} u^{\prime}(s) \phi(s) \psi(s) \mathrm{d} s \leq M \int_{t_{1}}^{t_{2}} \frac{u^{\prime}(s)}{(1+s)^{\gamma}} \mathrm{d} s \\
& =M\left(\int_{t_{1}}^{t_{2}}\left(\frac{u(s)}{(1+s)^{\gamma}}\right) \mathrm{d} s+\int_{t_{1}}^{t_{2}} \frac{\gamma u(s)}{(1+s)^{1+\gamma}} \mathrm{d} s\right) \\
& \leq M\left(\sup _{t \in[0,+\infty)} \frac{\beta_{2}(t)}{(1+t)^{\gamma}}-\inf _{t \in[0,+\infty)} \frac{\alpha_{1}(t)}{(1+t)^{\gamma}}+\sup _{t \in[0,+\infty)} \frac{\beta_{2}(t)}{1+t} \int_{0}^{+\infty} \frac{\gamma}{(1+s)^{\gamma}} \mathrm{d} s\right) \\
& \leq \int_{\eta}^{R} \frac{s}{h(s)} \mathrm{d} s
\end{aligned}
$$

which concludes that $u^{\prime}\left(t_{2}\right) \leq R$. For $t_{1}$ and $t_{2}$ are arbitrary, we obtain that if $u^{\prime}(t) \geq \eta$, then $u^{\prime}(t) \leq R, t \in[0,+\infty)$.

Similarly, we can also obtain that if $u^{\prime}\left(t_{1}\right)=-\eta, u^{\prime}(t)<-\eta, t \in\left(t_{1}, t_{2}\right]$, then $u^{\prime}(t) \geq-R$, $t \in[0,+\infty)$.

Step 3: (7) has at least three solutions. 
Define $T_{1}: X \rightarrow X$ as

$$
\left(T_{1} u\right)(t)=a C+B+C t+\int_{0}^{+\infty} G(t, s) \phi(s) f_{1}^{*}\left(s, u(s), u^{\prime}(s)\right) \mathrm{d} s
$$

where

$$
G(t, s)=\left\{\begin{array}{l}
a+s, 0 \leq s \leq t<+\infty \\
a+t, 0 \leq t \leq s<+\infty
\end{array}\right.
$$

Easily, the fixed point of $T_{1}$ coincides with the solution of BVP (7). It is enough to prove that $T_{1}$ has at least three fixed points.

With the similar discussions to those in [11], we can show that $T_{1}$ is completely continuous.

Let $N>\max \left\{\max \{C, a C+B\}+\max \{a, 1\} \cdot H_{R} \int_{0}^{+\infty} \phi(s) \psi(s) \mathrm{d} s,\left\|\alpha_{1}\right\|,\left\|\beta_{2}\right\|\right\}$, where $H_{R}$ $=\max _{0 \leq s \leq R} h(s)$. Set $\Omega=\left\{u \in X_{1},\|u\|<N\right\}$. Then for any $u \in \bar{\Omega}$, it holds

$$
\begin{aligned}
\left\|T_{1} u\right\| & =\max \left\{\left\|T_{1} u\right\|_{1},\left\|\left(T_{1} u\right)^{\prime}\right\|_{\infty}\right\} \\
& \leq \max \{C, a C+B\}+\max \{a, 1\} \int_{0}^{+\infty} \phi(s)\left|f_{1}^{*}\left(s, u(s), u^{\prime}(s)\right)\right| \mathrm{d} s \\
& \leq \max \{C, a C+B\}+\max \{a, 1\} H_{R} \int_{0}^{+\infty} \phi(s) \psi(s) \mathrm{d} s<N,
\end{aligned}
$$

so we obtain $\operatorname{deg}\left(I-T_{1}, \Omega, 0\right)=\operatorname{deg}(I, \Omega, 0)=1$. Let

$$
\begin{aligned}
& \Omega_{\alpha_{2}}=\left\{u \in \Omega, u(t)>\alpha_{2}(t), t \in[0,+\infty)\right\}, \\
& \Omega^{\beta_{1}}=\left\{u \in \Omega, u(t)<\beta_{1}(t), t \in[0,+\infty)\right\} .
\end{aligned}
$$

Because $\alpha_{2} \otimes \beta_{1}, \alpha_{1} \leq \alpha_{2} \leq \beta_{2}$ and $\alpha_{1} \leq \beta_{1} \leq \beta_{2}$, we have $\Omega_{\alpha_{2}} \neq \emptyset, \Omega \backslash \overline{\Omega_{\alpha_{2}} \cup \Omega^{\beta_{1}}} \neq \emptyset$ and $\Omega_{\alpha_{2}} \cap \Omega^{\beta_{2}}=\emptyset$. Noticing that $\alpha_{2}, \beta_{1}$ are strict lower and upper solutions, $T_{1}$ has no fixed point in the set $\partial \Omega_{\alpha_{2}} \cap \partial \Omega^{\beta_{2}}$. Therefore,

$$
\begin{aligned}
\operatorname{deg}\left(I-T_{1}, \Omega, 0\right) & =\operatorname{deg}\left(I-T_{1}, \Omega \backslash \overline{\Omega_{\alpha_{2}} \cup \Omega^{\beta_{2}}}, 0\right) \\
& +\operatorname{deg}\left(I-T_{1}, \Omega_{\alpha_{2}}, 0\right)+\operatorname{deg}\left(I-T_{1}, \Omega^{\beta_{1}}, 0\right) .
\end{aligned}
$$

In order to show $\operatorname{deg}\left(I-T_{1}, \Omega_{\alpha_{2}}, 0\right)=\operatorname{deg}\left(I-T_{1}, \Omega^{\beta_{1}}, 0\right)=1$, we define the operator $T_{2}: \bar{\Omega} \rightarrow \bar{\Omega}$ as

$$
\left(T_{2} u\right)(t)=a C+B+C t+\int_{0}^{+\infty} G(t, s) f_{2}^{*}\left(s, u(s), u^{\prime}(s)\right) \mathrm{d} s,
$$

where $f_{2}^{*}$ has the same expression as $f_{1}^{*}$ except changing $\alpha_{1}$ to $\alpha_{2}$. Similarly, we have $\alpha_{2}(t) \leq u(t) \leq \beta_{2}(t)$ for $t \in[0,+\infty)$ when $u$ is a fixed point of $T_{2}$. In another word, $\operatorname{deg}\left(I-T_{2}, \Omega \backslash \overline{\Omega_{\alpha_{2}}}, 0\right)=0$. Meanwhile, $T_{2} \bar{\Omega} \subset \bar{\Omega}$, so from Schäuder fixed point theorem, it holds 


$$
\operatorname{deg}\left(I-T_{2}, \Omega, 0\right)=1 .
$$

And we have

$$
\begin{aligned}
\operatorname{deg}\left(I-T_{1}, \Omega_{\alpha_{2}}, 0\right) & =\operatorname{deg}\left(I-T_{2}, \Omega_{\alpha_{2}}, 0\right) \\
& =\operatorname{deg}\left(I-T_{2}, \Omega, 0\right)-\operatorname{deg}\left(I-T_{2}, \Omega \backslash \overline{\Omega_{\alpha_{2}}}, 0\right) \\
& =1 .
\end{aligned}
$$

Similarly, we can deduce that $\operatorname{deg}\left(I-T_{1}, \Omega^{\beta_{1}}, 0\right)=1$. Therefore,

$$
\operatorname{deg}\left(I-T_{1}, \Omega \backslash \overline{\Omega_{\alpha_{2}} \cup \Omega^{\beta_{2}}}, 0\right)=-1 .
$$

Using the properties of the degree, we can obtain that $T_{1}$ has at least three fixed point $u_{1} \in \Omega_{\alpha_{2}}, u_{2} \in \Omega^{\beta_{1}}, u_{3} \in \Omega \backslash \overline{\Omega_{\alpha_{2}} \cup \Omega^{\beta_{1}}}$.

Remark 3.1. The strictness of the lower solution $\alpha_{2}$ and the upper one $\beta_{1}$ can be weakened, see [3].

If $f:[0,+\infty)^{3} \rightarrow[0,+\infty)$, we can establish a criteria for the existence of positive solutions.

Theorem 3.2. Let $f:[0,+\infty)^{3} \rightarrow[0,+\infty)$ be continuous and $\varphi \in L^{1}[0,+\infty)$. Suppose conditions $\left(\mathrm{H}_{1}\right)$ and $\left(\mathrm{H}_{3}\right)$ hold with $\alpha_{1}(t)>0, t \in(0,+\infty)$. Then, BVP (1) with $B, C \geq 0$ has at least three positive solutions satisfying the inequality (5).

Proof. Choose $R=\frac{1}{a}(B+\beta(0))$ and consider the boundary value problem (7) except $f_{R}$ substituting by

$$
f_{R}(t, x, y)=\left\{\begin{array}{l}
f(t, x, 0), y<0 \\
f(t, x, y), 0 \leq y \leq R \\
f(t, x, R), y>R
\end{array}\right.
$$

Similarly, we can obtain that the truncated problem has at least three solutions $u_{i}, i$ $=1,2,3$ satisfying $\alpha_{1}(t) \leq u_{i}(t) \leq \beta_{2}(t), t \in[0,+\infty)$. Because

$$
u_{i}^{\prime \prime}(t)=-\phi(t) f^{*}\left(t, u_{i}(t), u_{i}^{\prime}(t)\right) \leq 0
$$

and $u_{i}^{\prime}(+\infty)=C \geq 0$, we have

$$
0 \leq u_{i}^{\prime}(t) \leq u_{i}^{\prime}(0)=\frac{1}{a}\left(B+u_{i}(0)\right) \leq R .
$$

Consequently, $u_{i}, i=1,2,3$ are positive solutions of (1).

\section{Acknowledgements}

The authors would like to thank the referee for helpful comments and suggestions. This work was supported by the Fundamental Research Funds for the Central Universities (No. 2011YXL044) and the National Natural Science

Foundation of China (No. 111010385).

Received: 25 April 2011 Accepted: 30 November 2011 Published: 30 November 2011

\section{References}

1. Bai, ZB: The method of lower and upper solutions for a bending of an elastic beam equation. J Math Anal Appl. 248, 195-202 (2000). doi:10.1006/jmaa.2000.6887

2. Ehme, J, Eloe, PW, Henderson, J: Upper and lower solution methods for fully nonlinear boundary value problems. J Differ Equ. 180, 51-64 (2002). doi:10.1006/jdeq.2001.4056

3. Henderson, J, Thompson, HB: Existence of multiple solutions for second order boundary value problems. J Differ Equ. 166, 443-454 (2000). doi:10.1006/jdeq.2000.3797 
4. Rachunková, I: Upper and lower solutions and multiplicity results. J Math Anal Appl. 246, 446-464 (2000). doi:10.1006/ jmaa.2000.6798

5. $\mathrm{Xu}, \mathrm{X}$, Sun, JX: Solutions for an operator equation under the conditions of pairs of paralleled lower and upper solutions. Nonlinear Anal. 69, 2251-2266 (2008). doi:10.1016/..na.2007.08.005

6. Agarwal, RP, O'Regan, D: Infinite Interval Problems for Differential, Difference and Integral Equations. Kluwer Academic Publisher, Netherlands (2001)

7. Chen, S, Zhang, Y: Singular boundary value problems on a half-line. J Math Anal Appl. 195, 449-468 (1995). doi:10.1006/ jmaa.1995.1367

8. Agarwal, RP, O'Regan, D: Nonlinear boundary value problems on the semi-infinite interval: an upper and lower solution approach. Mathematika. 49, 129-140 (2002). doi:10.1112/S0025579300016120

9. Palamides, PK, Galanis, GN: Positive, unbounded and monotone solutions of the singular second Painlev equation on the half-line. Nonlinear Anal. 57, 401-419 (2004). doi:10.1016/j.na.2004.02.021

10. Yan, BQ, O'Regan, D, Agarwal, RP: Unbounded solutions for singular boundary value problems on the semi-infinite interval: upper and lower solutions and multiplicity. J Comput Appl Math. 197, 365-386 (2006). doi:10.1016/j. cam.2005.11.010

11. Lian, HR, Wang, PG, Ge, WG: Unbounded upper and lower solutions method for Sturm-Liouville boundary value problem on infinite intervals. Nonlinear Anal TMA. 70, 2627-2633 (2009). doi:10.1016/j.na.2008.03.049

12. Eloe, PW, Kaufmann, ER, Tisdell, CC: Multiple solutions of a boundary value problem on an unbounded domain. Dyn Syst Appl. 15(1), 53-63 (2006)

doi:10.1186/1687-2770-2011-51

Cite this article as: Lian and Geng: Multiple unbounded solutions for a boundary value problem on infinite intervals. Boundary Value Problems 2011 2011:51.

\section{Submit your manuscript to a SpringerOpen ${ }^{\circ}$} journal and benefit from:

Convenient online submission

- Rigorous peer review

- Immediate publication on acceptance

- Open access: articles freely available online

- High visibility within the field

- Retaining the copyright to your article

Submit your next manuscript at $\boldsymbol{s p r i n g e r o p e n . c o m ~}$ 